\title{
Model-based assessment of dynamic arterial blood volume flow from ultrasound measurements
}

\author{
C. A. D. Leguy $\cdot$ E. M. H. Bosboom • \\ A. P. G. Hoeks $\cdot$ F. N. van de Vosse
}

Published online: 6 May 2009

(c) The Author(s) 2009. This article is published with open access at Springerlink.com

\section{Erratum to: Med Biol Eng Comput \\ DOI 10.1007/s11517-009-0473-9}

Unfortunately, several errors occurred in Sect. 2.3.2 Statistical analysis, second paragraph.

The corrected paragraph is given below.

When considering a parameter $X$, the variability between the heartbeats of each measurement was evaluated by the intra-registration variability $\sigma_{h}$, which can be written as follows:

$\sigma_{h}=\sqrt{\frac{\sum_{v} \sum_{m} \sum_{b}\left(X_{v, m, b}-\bar{X}_{v, m}\right)^{2}}{\sum_{v} \sum_{m}\left(b_{v, m}\right)-m}}$

$X_{v, m, b}$ being the parameter value for the volunteer $v$, in measurement $m$ at heartbeat $b, \bar{X}_{v, m}$ the average parameter for measurement $m$ of volunteer $v$, and, $b_{v, m}$ and $m$ being the number of heart beats of the measurement $m$ for the volunteer $v$ and the total number of measurements, respectively.

The inter-registration variability $\sigma_{m}$ that evaluates the variability between the measurements of the volunteer can be written as:

$\sigma_{m}=\sqrt{\frac{\sum_{v} \sum_{m}\left(X_{v, m}-\bar{X}_{v}\right)^{2}}{\sum_{v}\left(m_{v}\right)-v} .}$

In this equation, $X_{v, m}$ is the parameter value of measurement $m$ for volunteer $v$ and $\bar{X}_{v}$ the average parameter for each volunteer $v$. The number of measurements for the volunteer $v$ and the number of volunteers are represented by $m_{v}$ and $v$ respectively.

The online version of the original article can be found under doi:10.1007/s11517-009-0473-9.

C. A. D. Leguy $(\bowtie) \cdot$ F. N. van de Vosse

Eindhoven University of Technology,

Eindhoven, The Netherlands

e-mail: c.a.d.leguy@tue.nl

E. M. H. Bosboom

Maastricht University Medical Centre,

Maastricht, The Netherlands

A. P. G. Hoeks

Cardiovascular Research Institute Maastricht,

Maastricht University, Maastricht, The Netherlands 